\title{
Methods of assessing late radiotherapy effects on bowel function
}

Authors:

Professor Annmarie Nelson, Marie Curie Research Centre, Division of Population Medicine, School of Medicine, 8th Floor Neuadd Meirionnydd, Heath Park, Cardiff University, CF14 4YS (NelsonA9@cardiff.ac.uk; 02920 687473)

Mala Mann, Specialist Unit for Review Evidence, Cardiff University

Professor John Staffurth, Division of Cancer and Genetics, School of Medicine, Cardiff University and Velindre University NHS Trust, Cardiff

\section{Acknowledgements:}

We would like to thank Elin Baddeley for her assistance with preparing the manuscript.

\section{Financial support ad sponsorship:}

AN and MM's posts are supported by Marie Curie Cancer Care core grant funding (grant reference: MCCC-FCO-11-C).

\section{Conflicts of interest:}

None

\section{Key messages:}

1. Pelvic radiation disease is under reported and under treated

2. Validated screening tools and treatment algorithms are available

3. Standardised outcome measures are needed to aggregate trial results and improve the quality of evidence

4. New radiation therapy techniques are developing to reduce harm to healthy tissue

5. Specialised services in gastroenterology departments are needed 
Abstract

Purpose: Pelvic Radiation Disease (PRD) in the form of chronic radiation induced consequences of treatment is under recognised by healthcare professionals and under reported by patients. Gastrointestinal symptoms are not routinely assessed, and may not be causally associated with previous radiotherapy. These symptoms are therefore often under treated.

Methods: A literature search was conducted in Ovid Medline, which included Epub Ahead of Print, In-Process \& Other Non-Indexed Citations and Ovid Embase for articles published between 2016 and April 2018. 11 papers were selected for review.

Results: A wide range of patient reported outcome measure instruments are used in research and practice. Clinical symptom grading is restricted to a fewer number of tools but may not always capture issues, such as urgency, that are important to the patient. Clinician and patient concordance in the assessment of outcomes is limited.

Initiatives to prompt communication of the relative risks of the late consequences of treatment and comparative effectiveness of treatments decisions are developing, as are new techniques to limit irradiation of healthy tissue.

Summary: Non-standardised outcome measurement reduces the ability to aggregate toxicity and patient outcomes across clinical trials. The development of standardised screening and treatment algorithms for gastrointestinal symptoms can systematically locate and treat gastrointestinal late effects of treatment.

Keywords: Radiotherapy, gastrointestinal, outcomes, screening, consequences of treatment. 
Radical or curative treatment has been delivered to around $2.5 \mathrm{~m}$ million people that have survived or are living with cancer in the UK currently, with this number predicted to increase to $4 \mathrm{~m}$ by 2030 [1]. Currently, around half of people with cancer have radiotherapy as part of their treatment [2] and of these, many will be facing consequential effects of their treatment. Symptoms may be acute and transitory or long term, sometimes appearing years later [3]. A recent James Lind Priority setting exercise focused on Living With and Beyond Cancer called for more research into the late consequential effects of cancer treatment as one of its top ten uncertainties, How can the short-term, long-term and late effects of cancer treatments be (a) prevented, and/or (b) best treated/ managed? [4].

Pelvic radiotherapy is used in the attempt to cure a range of cancers with an estimated figure of 17,000 patients receiving definitive or adjuvant pelvic radiotherapy per year in the UK [5]. Acute side effects of radiotherapy are common and most symptoms will fully resolve three months after the course of radiotherapy has been completed. Unfortunately, some people develop longterm symptoms after radiotherapy and these can be permanent; symptoms that develop more than three months after treatment are termed 'late effects', but if these are the continuation of acute effects that do not improve, they are termed 'consequential late effects'. The umbrella term of Pelvic Radiation Disease (PRD) has directed attention to this poorly understood area with an active patient group providing information and campaigning for greater awareness and research Pelvic Radiation Disease Association Additionally, Macmillan have published information extensively in this area with a programme dedicated to improving the outcomes for people suffering from late consequences of treatment.

It has been estimated that $90 \%$ of people experiencing PRD will develop a permanent change in their bowel habit, and $50 \%$ of all patients receiving pelvic radiotherapy experience a reduction in their quality of life $[6,7]$. In prostate cancer, approximately $40 \%$ of all treated patients state that 
the change in bowel function affects quality of life, and $20 \%$ or more say that this affects quality of life to a moderate or severe extent [7].

Bowel problems often lead to severe difficulties for cancer survivors (Figure 1) limiting travel, work and socialising. Patients may be too embarrassed to mention their symptoms or feel that they are an inevitable consequence of their treatment or age [8]. External beam radiation therapy (EBRT) treatment in particular is found to result in approximately half of patients having some form of bowel dysfunction; almost a third of those had consequential late effects following EBRT [9]

Figure 1: Word cloud of patient reported late effects following radiotherapy (EAGLE study data [50])

PRD in the form of chronic radiation-induced consequences of treatment is under recognised by healthcare professionals [6, 7], and under reported by patients [10]. Patients may believe that other clinical issues issues, such as whether their cancer has been cured, have priority in consultations, or are too embarrassed to raise the issue $[11,12]$. Other clinicians see little utility in identifying gastrointestinal symptoms, for example, assuming that few treatment options exist, as late radiation effects were always thought to be due to permanent fibrosis and be irreversible. [11,12]. Therefore, gastrointestinal symptoms are not routinely assessed and may not be causally associated with previous radiotherapy $[11,12]$. These symptoms are therefore often under treated, and are identified using different tools [10]. Combined with the use of nonvalidated tools, and limited patient reported outcomes, there is little opportunity to aggregate outcomes and results across trials [13].

This review will consider selected literature from 2016 to 2018 to understand the assessment and treatment of consequential effects of radiotherapy on bowel function. 


\section{Methods}

A literature search was conducted in Ovid Medline which included Epub Ahead of Print, In Process \& Other Non-Indexed Citations and Ovid Embase for articles published between 2016 and April 2018. The search strategy was designed using both text words and Medical subject headings (Table 1$)$.

Table 1: Search Strategy.

Sixty five records were screened after removing duplicates and irrelevant references. Thirty nine full-text articles assessed for eligibility and 11 papers of particular interest were included in the review (Figure 2). Of these, data from more than 20,000 people were included in 11 papers (Table 2) reporting randomised controlled trials or follow up results, reviews of the literature, and cohort studies. Radiation treatments included External Beam Radiation Therapy (EBRT), External Megavoltage Radiotherapy (EMRT), Brachytherapy, hypofractionated IMRT (HIMRT), Hypofractionated High Dose Intensity Modulated Radiotherapy (HFRT), Stereotactic Ablative Radiotherapy (SABR), and Proton Therapy (PT).

Figure 2. Study flow Diagram

\section{Overview of selected papers}

Within the selected literature, 9 of 11 papers focused on prostate cancer, perhaps not surprisingly, given the high incidence, its large surviving population, and high number of clinical trials. Rectal and colorectal cancer were also included. The range of follow-up periods to assess consequences of treatments ranged between one year to ten years, where this was stated. 
A range of study designs are included, CTIMPs, RCTs and follow up reporting, cohort and observational, registry data or narrative literature review. Interventions include medicinal products, dose escalation, new radiation therapies and novel techniques.

Table 2. Included papers.

The papers report toxicities outcomes following radiation therapy using a range of measures (Table 3), including clinician assessed grading of symptoms LENT/SOMA [14, 15], CTCAE [16] and RTOG [17] and patient reported outcome measures, including IPSS [18], EPIC [19], QLQ C30 [20], QLQ PR25 [21], FACT-G [22], SF12 [23], EPIC 26 [24]).

Some studies did not specify patient outcome measures [25] or used non validated PROMS $(* * 26)$ or pragmatic data collection methods including non-validated questionnaires. One study constructed a single question 'overall how much does your bowel function affect your quality of life [**27].

Five studies used both clinician and patient reported outcome measures to assess symptoms $[25, * 28, * 29,30, * * 31]$, the remainder reported patient reported outcomes only.

Table 3. Symptom and QoL measurement tools.

\section{Risk assessment, comparative effectiveness and treatment decision-making}

Two papers paid attention to aspects relating to treatment decision-making via the competing risks of treatments, including late effects [**26], or how treatment decisions were made [**27]. Both papers explored how the potential for late effects and comparative harms of treatment modalities is communicated to the patient $[* * 26, * * 27]$. Suggested interventions included pretreatment counselling regarding risk of harm, and use of a nomog raphic consent aid, designed by patients, showing the predicted percentages of affected bowel function according to 
associated variables of disease and treatment. However, in a narrative synthesis of trials of interventions for prostate cancer, it was acknowledged that the evidence base for the clinical effectiveness of treatments, including surgery or radiotherapy, is weak due to underpowered clinical trials and differing assessment measures, thereby further complicating treatment decision-making [*32]. In a narrative synthesis of the literature looking at quality of life outcomes following a broader range of treatments for prostate cancer (including active surveillance (AS)), the research team focused on validated patient reported outcome measures (PROMS) exclusively [*33]. The study confirms similar outcomes [*32] with reported QoL issues specific to treatment modalities: surgery/urosexual symptoms, EBRT/ bowel symptoms, Brachytherapy/short term urinary symptoms, whilst AS is associated with high scores for QoL.

In terms of concordance of clinician vs patient reported outcomes, one paper noted the disparity between the clinician and patient assessments [*29] (albeit using a non-validated questionnaire), especially in relation to urgency, which was noted as an enduring effect observed in 7\% of men at three years post EBRT for prostate cancer [15].

Given the lack of aggregated outcome sets, and in support of treatment decision -making with regard to QoL, a group based in Melbourne have established a clinical registry to collect and analyse patient-centred outcomes [**31]. Men with prostate cancer are routinely telephoned about health outcomes and QoL using questions from validated QoL tools at 12 and 24 months from diagnosis. An analysis of patient data from 1825 men revealed outcome differences between treatment centres enabling a further evaluation of centres not matching the standard of others.

\section{Limiting effects of treatment}

The incidence and grade of gastrointestinal toxicity is associated with radiation dose and volume of irradiated healthy bowel tissue [3] and the importance of reducing toxicities of treatment and preserving QoL are key concerns in managing existing treatments or developing new treatments. 
These include new techniques of targeted radiation therapy, which aims to reduce the volume of sensitive normal tissues being irradiated, and trials of drugs that may protect healthy tissue have been reported recently.

Some populations may be under represented in clinical trials, a lack of racial diversity [**26] or a narrow age range increases the risk of trials bias or incorrect assumptions about treatment tolerance. The $\mathrm{CHHiP}$ trial found that hypofractionated high dose intensity modulated radiation therapy for prostate cancer is non inferior to conventional treatment. In an exploratory analysis and in an exploratory analysis [*34], reported on the outcomes of 491 men aged 75+, constituting $15 \%$ of the trial participants, and concluded that the treatments were well tolerated and that age should not be a barrier to treatment. Of interest is the fact that patient reported outcomes increased the reporting of bowel bother, which were not reported in the outcomes measured by validated symptom questionnaires, including RTOG and LENT-SOMA.

Proton beam therapy (PBT) may result in less damage to surrounding tissue due to targeted treatment and lack of exit dose but has a limited evidence base in terms of patient outcomes. One treatment centre has retrospectively reviewed its first 100 patients receiving PBT for prostate cancer [ $\left.{ }^{*} 28\right]$. Late outcomes for 79 patients were reported after a minimum of three months using clinical grading (CTCAE v.4) [35] in combination with PROMS (IPSS and EPIC). Both sets of outcomes compare favourably to photon therapy but the evidence is limited due to the small, heterogenous study population.

In a phase II randomised controlled trial of treatment delivery with the use of a hydrogel spacer to protect the rectal wall, GI toxicities were reduced and QoL scores were improved after five years in 54 patients with prostate cancer [*36]. However, for an NHS setting, the high cost of injections may be prohibitive.

Despite advances in treatment dose distribution and techniques, damage to rectal tissue still occurs. A different approach sees the potential for protection of the tissue using Lovastatin, a 
drug which has the ability to protect mice from the late effects of radiation treatment.

Unfortunately, when tested in a non randomised trial of 53 adults with prostate cancer, it was not effective [25].

\section{Conclusion}

Communication of the risk of late effects of radiation therapy is increasingly complex.

Gastrointestinal late effects can be debilitating and in the interests of patient-centred health care, communication of potential risk is essential to shared decision-making. Additionally, due to the lack of a standardised approach to measuring patient outcomes, aggregation of data across clinical trials and clinical settings is not feasible, leading to low evidence of the toxicities associated with radiation therapy interventions.

New techniques to improve the preservation of healthy tissue are developing rapidly, including focal salvage for prostate cancer, which aims to avoid whole gland exposure to radiation, with tentative evidence that GI and erectile toxicities are reduced [30]. However, awareness of the potential for consequences of treatment is essential. Late radiation effects may be perceived by clinicians as irreversible, with limited treatment options. Clinicians may not actively seek information on late radiation effects, focussing primarily on symptoms of cancer [11, 12]. Patients, viewing symptoms as expected consequences of therapy, or being too embarrassed to mention them, or grateful for curative treatment may not volunteer their symptoms during consultations, regardless of the ongoing effects on their physical, psychological and social wellbeing. Enabling patients to report symptoms as a matter of course and consistent assessment by clinicians, is key to fully understanding the extent of the issue, and ultimately improving long term patient care.

For a screening tool to be effective in a busy clinical setting, the questionnaire should be succinct and simple. The Late Effects on Normal Tissue Subjective, Objective, Management, and Analytic (LENT-SOMA) questionnaire (now largely superseded by Common Terminology Criteria 
for Adverse Events [CTCAE] [16], was developed to assess a wide range of problems after radiotherapy [37]. However, despite efforts to reduce the length of this questionnaire [38], it is too long to be used routinely as a screening tool [37]. Other symptom grading scoring systems are also either too long (e.g. the modified Inflammatory Bowel Disease Questionnaire [IBDQ] [39] with 32 items and the Gastrointestinal Symptom Rating Scale [GSRS] [39, 40, 41, 42, 43] with 15 items, or they focus on just one symptom (e.g. the Vaizey Incontinence Questionnaire with seven items focussing on bowel continence) [42]. Therefore, there is a need for a simple screening tool that can be used in clinical practice.

\section{Screening}

ALERT- B (Figure 3) is a three question validated tool that can effectively detect patients with ongoing gastrointestinal symptoms that have developed after pelvic radiotherapy for cancer [44]. The tool is specified in the latest version of the Royal College of Radiologists 'Modernising radiotherapy services in England' (forthcoming) and can be used at any time post pelvic radiation therapy.

Figure 3: Alert-B Screening Tool questions

\section{Treatment}

Research carried out at the Royal Marsden Hospital (RMH), has identified 23 symptoms that frequently occur after prostate radiotherapy $[11,12]$. A sophisticated algorithm has been developed that allows these symptoms to be managed in a systematic way [45] and tested in The Optimising Radiotherapy Bowel Injury Therapy (ORBIT) trial for pelvic cancer (prostate, bladder, vulvar, vaginal cervical, endometrial, anal and rectal cancer) $[45,46,47,48]$. The trial has shown that these symptoms can be accurately diagnosed and effectively treated at low cost with high benefit to the patient $[46,47]$. The ORBIT trial has also demonstrated that specialist 
trained nurses can deliver this assessment and treatment as effectively as a doctor in most patients. Other centres trialling the algorithm have also found it to effectively improve patients' symptoms at modest cost $[49,50]$ and have highlighted the need for specialized services embedded within gastroenterology.

Late effects of treatment can occur months or years later, which emphasises the need for consistent and timely assessments at key points. Given that many patients may be lost to follow up whilst there symptoms occur, one solution would be to place an assessment directly in the hands of the patient. The Alert B tool is currently being tested as a patient reported outcome measure available on a commercial electronic patient platform. This work is ongoing in England and Wales as a collaboration between Macmillan and the Royal College of Radiologists.

PRD is an under recognised and under treated consequence of radiation therapy. However, ongoing work to screen and treat gastrointestinal symptoms, and the development of new techniques to limit damage to healthy tissue during radiation therapy will increase awareness and support a reduction in the incidence of this distressing condition. 


\section{References}

[1] Maddams, J., Utley, M., \& Møller, H. (2012). Projections of cancer prevalence in the United Kingdom, 2010-2040. British journal of cancer, 107(7), 1195.

[2] Cancer Research UK. What is radiotherapy? Retrieved 17/01/2019, 2019, from https://www.cancerresearchuk.org/about-cancer/cancer-ingeneral/treatment/radiotherapy/about

[3] Frazzoni, L., La Marca, M., Guido, A. et al. Pelvic radiation disease: Updates on treatment options. World J Clin Oncol. 2015;6(6):272-80.

[4] National Cancer Research institute (NCRI) Living with and beyond cancer. Retrieved from http://www.ncri.org.uk/wp-content/uploads/2018/11/UK-Top-living-with-and-beyond-cancerresearch-priorities.pdf 02/02/19

[5] Henson, C.C., Andreyev, H.J., Symonds, R.P. et al. (2011). Late-onset bowel dysfunction after pelvic radiotherapy: a national survey of current practice and opinions of clinical oncologists. Clinical Oncology, 23(8), 552-557.

[6] Andreyev, H. (2007). Gastrointestinal problems after pelvic radiotherapy: the past, the present and the future. Clinical Oncology, 19(10), 790-799.

[7] Andreyev, J. (2007). Gastrointestinal symptoms after pelvic radiotherapy: a new understanding to improve management of symptomatic patients. The lancet oncology, 8(11), 1007-1017.

[8] Abayomi, J., Kirwan, J., \& Hackett, A. (2009). The prevalence of chronic radiation enteritis following radiotherapy for cervical or endometrial cancer and its impact on quality of life. European Journal of Oncology Nursing, 13(4), 262-267. 
[9] Lehto, U.-S., Tenhola, H., Taari, K., \& Aromaa, A. (2017). Patients' perceptions of the negative effects following different prostate cancer treatments and the impact on psychological wellbeing: a nationwide survey. British journal of cancer, 116(7), 864.

[10] Henson, C.C., Davidson, S.E., Ang, Y. et al. (2013). Structured gastroenterological intervention and improved outcome for patients with chronic gastrointestinal symptoms following pelvic radiotherapy. Supportive Care in Cancer, 21(8), 2255-2265.

[11] Andreyev, H.J.N., Vlavianos, P., Blake, P. et al. (2005). Gastrointestinal symptoms after pelvic radiotherapy: role for the gastroenterologist? International Journal of Radiation Oncology* Biology* Physics, 62(5), 1464-1471.

[12] Andreyev, J. (2005). Gastrointestinal complications of pelvic radiotherapy: are they of any importance? Gut, 54(8), 1051-1054.

[13] Heneghan, C., Goldacre, B. \& Mahtani, K.R. Why clinical trial outcomes fail to translate into benefits for patients. Trials 2017; 18: 122.

[14] Pavy, J.J., Denekamp, J., Letschert, J. et al. (1995). Late effects toxicity scoring: The SOMA scale. Radiother Oncol 1995;35:11-15.Pelvic Radiation Disease Association. We can help Retrieved 17/01/2019, 2019, from http://www.prda.org.uk/

[15] Rubin, P., Constine, L.S., Farjardo, L.F. et al. (1995). Overview of Late Effects Normal Tissues (LENT) scoring system. Radiother Oncol 1995;35:9-10.

[16] National Cancer Institute. (2015). Common Terminology Criteria for Adverse Events (CTCAE) Retrieved 17/01/2019, 2019, from https://ctep.cancer.gov/protocoldevelopment/electronic_applications/docs/ctcae_v5_quick_ref erence_8.5x11.pdf 
[17] Radiation Therapy Oncology Group. Late Radiation Morbidity Scoring Schema https://www.rtog.org/ResearchAssociates/AdverseEventReporting/CooperativeGroupCommonT oxicityCriteria.aspx last retrieved 06/02/19

[18] Barry, M.J., Fowler, F.J. Jr., O'Leary, M.P. et al. (1992). The American Urological Association symptom index for benign prostatic hyperplasia. The Measurement Committee of the American Urological Association. J Urol. 1992 Nov;148(5):1549-57; discussion 1564.

[19] Twei, J., Dunn, R.L., Litwin, M.S. et al. (2000). Development and validation of the expanded prostate cancer index composite (EPIC) for comprehensive assessment of health-related quality of life in men with prostate cancer. Urology, Vol.56(6), pp.899-905.

[20] Aaronson, N.K., Ahmedzai, S., Bergman, B. et al. (1993) The European Organization for Research and Treatment of Cancer QLQ-C30: a quality-of-life instrument for use in international clinical trials in oncology. J.Natl.Cancer Inst., 85, 365-376.

[21] Van Andel, G., Bottomley, A., Fossa, S. et al. (2008). An international field study of the EORTC QLQ-PR25: A questionnaire for assessing the health related quality of life of patients with prostate cancer. European Journal of Cancer, 44(16), 2418-2424.

[22] Cella D.F., Tulsky D.S., Gray G. et al. (1993). The Functional Assessment of Cancer Therapy scale: development and validation of the general measure. J Clin Oncol. 1993 Mar;11(3):570-9. [23] Ware, J. Jr., Kosinski, M., Keller, SD. (1996). A 12-Item Short-Form Health Survey: construction of scales and preliminary tests of reliability and validity. Med Care. 1996 Mar;34(3):220-33.

[24] Szymanski, K.M., Weic, J.T., Dunn, R.L., \& Sand, M.G. (2010) Development and Validation of an Abbreviated Version of the Expanded Prostate Cancer Index Composite Instrument (EPIC-26) for Measuring Health-Related Quality of Life Among Prostate Cancer Survivors. Urology. Vol.76(5): 1245-1250. 
[25] Anscher, M.S., Chang, M.G., Moghanaki, D., Rosu, M. et al (2018). A Phase II Study to Prevent Radiation-induced Rectal Injury With Lovastatin. American Journal of Clinical Oncology. $41(6): 544-548$.

[**26] Barocas, D.A., Alvarez, J., Resnick, M.J. et al. (2017). Association Between Radiation Therapy, Surgery, or Observation for Localized Prostate Cancer and Patient-Reported Outcomes After 3 Years. JAMA, Vol.317(11), pp.1126-1140.

[**27] Battersby NJ, Bouliotis G, Emmertsen KJ on behalf of the UK and Danish LARS Study Groups, et al. Development and external validation of a nomogram and online tool to predict bowel dysfunction following restorative rectal cancer resection: the POLARS score. Gut 2018;67:688-696.

[*28] Deville Jr, C., Jain, A., Hwang, W. et al. (2018). Initial report of the genitourinary and gastrointestinal toxicity of post-prostatectomy proton therapy for prostate cancer patients undergoing adjuvant or salvage radiotherapy. Journal Acta Oncologica, Vol.57(11), pp.15061514.

[*29] Hoffman, K.E., Skinner, H., Pugh, T.J. et al. (2018). Patient-reported Urinary, Bowel, and Sexual Function After Hypofractionated Intensity-modulated Radiation Therapy for Prostate Cancer: Results From a Randomized Trial. American journal of clinical oncology, Vol.41(6), pp.558-567.

[30] Duijzentkunst, D., Peters, M., Voort van Zyp, J., Moerland, M. et al. (2016). Focal salvage therapy for local prostate cancer recurrences after primary radiotherapy: a comprehensive review. World Journal of Urology, Vol.34(11), pp.1521-1531

[**31] Bandarage, V.K.P, Billah, B., Millar, J.L., \& Evans, S. (2016). Prospective evaluation of patient-reported quality of life outcomes after external beam radiation treatment for prostate 
cancer in Victoria: A cohort study by the Victorian Prostate Cancer Registry. Journal of Medical Imaging and Radiation Oncology, Vol.60(3), pp.420-427.

[*32] Wallis, C.J.D., Glaser, A., Hu, J.C. et al. (2017). Survival and Complications Following Surgery and Radiation for Localized Prostate Cancer: An International Collaborative Review. European Urology, Vol.73(1), pp.11-20.

[*33] Lardas, M., Liew, M., Van Den Bergh, R.C. et al. (2017). Quality of Life Outcomes after Primary Treatment for Clinically Localised Prostate Cancer: A Systematic Review. European Urology, Vol.72(6), pp.869-885.

[*34] Wilson, J.M., Dearnaley, D.P., Syndikus, I. et al. (2018). The Efficacy and Safety of Conventional and Hypofractionated High-Dose Radiation Therapy for Prostate Cancer in an Elderly Population: A Subgroup Analysis of the CHHiP Trial. International Journal of Radiation Oncology*Biology*Physics, Vol.100(5), pp.1179-1189.

[35] U.S.DEPARTMENT OF HEALTH AND HUMAN SERVICES, National Institutes of Health, National Cancer Institute. CTCAE v 4 - Common Terminology Criteria for Adverse Events (CTCAE) Version 4.0. (2009). V4.03: June 14, 2010).

[*36] Pinkawa, M., Berneking, V., Schlenter, M. et al. (2017). Quality of Life After Radiation Therapy for Prostate Cancer With a Hydrogel Spacer: 5-Year Results. Int J Radiat Oncol Biol Phys, 1;99 (2): (374-377).

[37] Seegenschmiedt, M. (1995). 16.1 Supportive Maßnahmen in der Strahlentherapie der Haut: Literatur. Radiother Oncol, 35, 17-60.

[38] Farnell, D.J., Mandall, P., Anandadas, C. et al. (2010). Development of a patient-reported questionnaire for collecting toxicity data following prostate brachytherapy. Radiotherapy and Oncology, 97(1), 136-142. 
[39] Guyatt, G., Mitchell, A., Irvine, E. J. et al. (1989). A new measure of health status for clinical trials in inflammatory bowel disease. Gastroenterology, 96(3), 804-810.

[40] Blair, J., Conrad, F., Ackermann, A.C., \& Claxton, G. (2006). The effect of sample size on cognitive interview findings. Paper presented at the Proceedings of the American Statistical Association.

[41] Kulich, K.R., Madisch, A., Pacini, F. et al. (2008). Reliability and validity of the Gastrointestinal Symptom Rating Scale (GSRS) and Quality of Life in Reflux and Dyspepsia (QOLRAD) questionnaire in dyspepsia: a six-country study. Health and quality of life outcomes, 6(1), 1.

[42] Vaizey, C., Carapeti, E., Cahill, J., \& Kamm, M. (1999). Prospective comparison of faecal incontinence grading systems. Gut, 44(1), 77-80.

[43] Willis, G. B. (2004). Cognitive interviewing: A tool for improving questionnaire design: Sage Publications.

[44] Taylor, S., Byrne, A., Adams, R. et al. (2016) The three item ALERT-B questionnaire provides a validated screening tool to detect chronic gastrointestinal symptoms after pelvic radiotherapy in cancer survivors. Clin Oncol 2016;28:e139-47. doi: 10.1016/j.clon.2016.06.004. Epub 2016 Jun 29

[45] Andreyev, H.J.N., Benton, B. E., Lalji, A. et al. (2013). Algorithm-based management of patients with gastrointestinal symptoms in patients after pelvic radiation treatment (ORBIT): a randomised controlled trial. The Lancet, 382(9910), 2084-2092.

[46] Andreyev, H., Thomas, K., Benton, B. et al. (2012). OC-083 Optimising radiation bowel injury therapy, the orbit study, a randomised controlled trial. Gut, 61(Suppl 2), A36-A36. 
[47] Andreyev, H.J.N., Davidson, S.E., Gillespie, C. et al. (2012). Practice guidance on the management of acute and chronic gastrointestinal problems arising as a result of treatment for cancer. Gut, 61(2), 179-192.

[48] Benton, B., Norton, C., Lindsay, J. et al. (2011). Can nurses manage gastrointestinal symptoms arising from pelvic radiation disease? Clinical oncology, 23(8), 538-551.

[49] Taylor, S., Demeyin, W., Muls, A. et al. (2016). Improving the Wellbeing of Men by Evaluating and Addressing the Gastrointestinal Late Effects of Radical Treatment for Prostate Cancer (EAGLE): study protocol for a mixed-method implementation project. BMJ Open 2016;6:e011773. doi: 10.1136/bmjopen-2016-011773

[50] Staffurth, J., Sivell, S., Ahmedzai, S. et al. (2018). The Impact of Specialized Gastroenterology Services for Late Pelvic Radiation Disease: Results from the Prospective Multicenter EAGLE Study. International Journal of Radiation Oncology*Biology*Physics Vol.102(3), Supplement, 1 November 2018, Page S35. 